\title{
In Vitro and In Vivo Enhancement of Antitumoral Activity of Liposomal Antisense Oligonucleotides by Cineole as a Chemical Penetration Enhancer
}

\author{
Hamid Reza Moghimi, ${ }^{1}$ Farshad H. Shirazi, ${ }^{1}$ Mehdi Shafiee Ardestani, \\ Mohammad Ali Oghabian, ${ }^{3}$ Mostafa Saffari, ${ }^{4,5}$ and Jafar Sojoudi ${ }^{6}$ \\ ${ }^{1}$ School of Pharmacy, Shahid Beheshti University of Medical Sciences, Tehran, Iran \\ ${ }^{2}$ Radiopharmacy Department, Tehran University of Medical Sciences, Tehran, Iran \\ ${ }^{3}$ Research Center for Science and Technology in Medicine, Tehran University of Medical Sciences, Tehran, Iran \\ ${ }^{4}$ School of Pharmacy, Iran University of Medical Sciences, Tehran, Iran \\ ${ }^{5}$ Department of Medical Nanotechnology, Faculty of Advanced Sciences \& Technology, Pharmaceutical Sciences Branch, \\ Islamic Azad University (IAUPS), Tehran, Iran \\ ${ }^{6}$ Pharma Chemie Company, Tehran, Iran
}

Correspondence should be addressed to Mostafa Saffari; mostafa.saffary@gmail.com

Received 6 July 2015; Revised 18 August 2015; Accepted 19 August 2015

Academic Editor: Abdelwahab Omri

\begin{abstract}
Copyright (C) 2015 Hamid Reza Moghimi et al. This is an open access article distributed under the Creative Commons Attribution License, which permits unrestricted use, distribution, and reproduction in any medium, provided the original work is properly cited.

Cellular uptake and cytoplasmic release of liposomal antisense oligonucleotides (AsODNs), which can act as rate-limiting steps, are still remained to be completely optimized. Here, the possibility of enhancing such processes at cellular and animal levels by cineole, as a penetration enhancer, was investigated. A cationic nanoliposome containing an AsODN against PKC- $\alpha$ and a cineolecontaining nanoliposome were prepared and characterized. The effect of nanoliposomal cineole on sequence-specific cytotoxicity of nanoliposomal AsODN against A549, was studied in vitro (MTT, flow cytometry, fluorescence microscopy, and real time PCR) and in vivo (xenograft lung tumor in nude mice) using different concentrations and treatment times. Results showed specific cytotoxicity of nanoliposomal AsODN was increased significantly from $11 \%$ to $25 \%$ when A549 cells were exposed to $10 \mu \mathrm{g} / \mathrm{mL}$ cineole for 1 or 4 hours. This inhibitory effect was further increased to about $40 \%$ when the concentration was increased to $40 \mu \mathrm{g} / \mathrm{mL}$ for 1 hour. In animal studies, cineole significantly decreased the tumor volume (about 75\%) and increased its doubling time from 13 days to 31 days. A linear relationship exists between cineole concentration and its enhancement effects. Finally it was concluded that cineole, and possibly other membrane fluidizers, can improve nanoliposomal gene therapy at cellular and animal levels.
\end{abstract}

\section{Introduction}

Antisense oligonucleotides (AsODNs), targeting specific gene or mRNA, are capable of potently downregulating proliferation and invasion in human cancer cells. They mainly act through RNAse-H activation or hybrid arrest (i.e., steric blockage of translation) [1, 2]. Being polyanionic and large, AsODNs practically are unable to permeate cell membranes well and, therefore, they have compromised biological activity. Rationally designed carriers can help to overcome these restrictions [3].
Cationic nanoliposomes are among the most widely used nonviral carriers for macromolecules. They encapsulate nucleic acids efficiently through electrostatic interaction and condense them to form complexes called lipoplexes [4]. Liposomal encapsulation of AsODNs improves their transfection efficiency and increases their resistance to nuclease degradation $[5,6]$. These carriers are mainly internalized into the target cells through endocytosis [7-9]. Afterward, AsODNs have to be released from endosomal compartment into cytoplasm through membrane destabilization and fusion mechanisms. However, lysosomal degradation of AsODNs 
can occur especially if AsODNs fail to be released from endosomes at early stages as $\mathrm{pH}$ falls in endosomal compartment later $[10,11]$. After release, AsODNs may act in the cytoplasm or may migrate from cytoplasm into nucleus [12].

Among the above-mentioned steps, cellular uptake and release from endosomes are the most significant barriers in antisense therapy $[13,14]$. To overcome these barriers, different strategies have been employed such as incorporating nonbilayer forming lipids (e.g., DOPE) into liposomes, application of cell penetrating peptides (CPPs) [15-19], or employment of physical methods such as ultrasound [20] that somehow are difficult to be employed. However, despite all of these efforts, the problem has remained to be completely solved by a safe, easy, and economic method.

On the other hand, there are several molecules, called penetration enhancers, that are widely used to diminish the barrier function of different biological membranes (e.g., stratum corneum in skin) to facilitate drug delivery to and through the tissue [21]. Some of these molecules work by disruption of lipid bilayers, which are also present in the liposomes, cell membrane, and endosomes. Therefore, it was decided here to investigate the application of such membrane fluidizing agents (penetration enhancers) as a novel strategy for improving delivery of liposomal ODN, in both in vitro (cell lines) and in vivo (animal) models. Among different chemical penetration enhancers is cineole, which acts mainly through lipid bilayer fluidization and its mechanism of action is well documented [22-24] and was chosen for the present investigation.

Cineole, the major component of eucalyptus oil, belongs to one of the biggest class of skin penetration enhancers (i.e., terpenes) and has been shown to increase permeation of hydrophilic and lipophilic drugs through human skin [2, 25], lamellar model structures [22, 26], and rat skin [27]. Its mechanisms of action are said to be extraction of lipids and keratin denaturation [28], increase in fluidity of lamellar membranes $[22,29]$, and improving drug partitioning to core of lamellar membranes $[23,30]$, most of which are expected to act on liposomes.

In this investigation, based on earlier survey, PKC- $\alpha$ as an impressive inhibitory target for gene therapy in adenocarcinoma cells was selected. The impact of cineole on efficiency of liposomal AsODNs against protein kinase C- $\alpha$ (PKC- $\alpha$ ) was evaluated through studying molecular transport and viability of human non-small-cell lung adenocarcinoma (A549) cells and suppression of tumor growth in A549 xenograft model in nude mice [31].

\section{Experimental Section}

2.1. Materials. DOTAP, PEG2000-DSPE, egg PC were obtained from Lipoid GMBH (Ludwigshafen, Germany). DSPC was purchased from Northern Lipids (Vancouver, Canada). Cholesterol (Chol) was purchased from Sigma Chemical Company (St. Louis, MO). A 20-mer phosphorothioate oligodeoxynucleotide, $5^{\prime}$-TsCsCs AsTsGs AsCsGs AsAsGs TsAsCs AsGsCs CsGs-3' (AsODN) directed against PKC- $\alpha$ mRNA, and its random or scrambled sequence (ScODN), $5^{\prime}$-CsGsAs GsCsAs CsGsCs AsGsTs AsTsCs
AsCsTs AsGs-3', that was used as a control oligonucleotide $(\mathrm{ScODN})$ were synthesized by Bioneer (Korea) [31]. Fluorescein isothiocyanate tagged phosphorothioate oligonucleotide (FITC-ON) was synthesized and purified by Synthegen (Houston, TX, USA). Fetal bovine serum, Dulbecco's modified Eagle's medium, RPMI 1640 medium, trypan blue stain, and trypsin-EDTA solution were purchased from Gibco BRL. 1,8 cineole (>99\%) and MTT were obtained from Sigma Chemical Company (St. Louis, MO). All other used reagents were of analytical grade.

\subsection{Methods}

2.2.1. Preparation and Characterization of Cationic Liposomes. PEG stabilized ODN-encapsulated liposomes (also called stabilized antisense lipid particles or SALPs) were prepared by the method described previously $[6,32]$. Briefly, ethanolic lipid solution was injected into ODN solution $(10 \mu \mathrm{M})$ in citrate buffer $(\mathrm{pH}=4)$. Lipid mixture was composed of DSPC/Chol/PEG lipid/DOTAP (20: $45: 10: 25$, mol \%). Dialysis was performed against citrate buffer for 2 hours and subsequently HEPES buffered saline (HBS, $\mathrm{pH}=7.4$ ) overnight. DEAE-Sepharose CL-6B gel chromatography column was prepared according to the instructions of the manufacturer and unencapsulated ODN was removed using this anionexchanger resin. The nanoliposomes were then stored at $4^{\circ} \mathrm{C}$ until use within one month.

Particle size and zeta-potential of SALPs were determined by Malvern Zetasizer (UK). ODN content was measured by spectrophotometry (Shimadzu, Japan) after solubilizing nanoliposomes [32] and phospholipid content was determined after Bligh and Dyer lipid extraction [33] by Stewart methods [34] (three replicates each). Nanoliposomal encapsulation efficiency at any step was then calculated using

$$
\mathrm{EE} \%=\left[\frac{(\mathrm{C} 2 / \mathrm{L} 2)}{(\mathrm{C} 1 / \mathrm{L} 1)}\right] \times 100,
$$

where C2 and L2 are ODN and lipid content of nanoliposomes, at any given stage, respectively, and $\mathrm{C} 1$ and $\mathrm{L} 1$ are, respectively, initial ODN and lipid content of nanoliposomes.

\subsubsection{Preparation of Liposomal Cineole by Thin Layer Film} Hydration. Cineole is a lipophilic compound and it is not possible to deliver it by simple aqueous solution. For in vitro application of such compounds, DMSO or other organic solvents are usually used to solve material in culture media. For in vivo purpose (especially in our survey), however, it is better to avoid organic solvents due to their cytocidal effect and interference in experimental results [35]. As advantages of liposome as vehicle for essential oils have been shown [36], it was decided here to formulate cineole as nanoliposomes.

Cineole-containing nanoliposomes were prepared by thin film hydration method with modification of Sinico method [36]. Briefly egg phosphatidylcholine (egg PC)/DOTAP/chol/ cineole (14:1:7:78 mol\%) were dissolved in $10 \mathrm{~mL}$ chloroform and the solvent was evaporated in a rotary balloon and shaked for 2 hours at $40^{\circ} \mathrm{C}$. The obtained lipid film was then hydrated with HBS ( $\mathrm{pH}$ 7.4) to form initial nanoliposomes at $40^{\circ} \mathrm{C}$. Nanoliposomes were then extruded (3 times through 
$200 \mathrm{~nm}$ and 3 times through $100 \mathrm{~nm}$ ) polycarbonate filter membranes (Millipore, USA) and subsequently purified by G50 Sephadex column to separate free cineole.

2.2.3. Cineole Assay and Encapsulation Efficiency. Cineolecontaining nanoliposomes were destroyed in an equal volume of methanol to give a clear solution and the solution was then assayed for cineole by a GC method using a Shimadzu gas chromatography system using BPX1 column and connected to FID detector (Shimadzu Co., Japan). This method employed a calibration curve in the range of 0.03 to $10 \mathrm{mg} / \mathrm{mL}$ using solution of cineole in water/methanol (50:50), $n=3$. The encapsulation efficiency (EE \%) of liposomal cineole was calculated by (1).

2.2.4. Cell Culture Studies. A549 cells, obtained from Pasteur Institute (Tehran, Iran), were grown in RPMI 1640 medium supplemented with $10 \%$ heat-inactivated FBS, $100 \mathrm{U} / \mathrm{mL}$ penicillin, and $100 \mu \mathrm{g} / \mathrm{mL}$ streptomycin. They were maintained at $37^{\circ} \mathrm{C}$ in a $5 \% \mathrm{CO}_{2}$-incubator (Heraeus, Germany). Cell viability was evaluated either by cell count using trypan blue stain $0.4 \%$ under inverted microscope (Leica, Germany) or by MTT assay [37].

To determine enhancer dose, the cytotoxic effect of liposomal cineole on cells was first evaluated at 1 to $40 \mu \mathrm{g} / \mathrm{mL}$ cineole concentration and incubation time of 1 and 4 hours followed by cell viability evaluation after 48 hours by MTT assay.

For enhancement studies, the cells were first treated for 1 or 4 hours with nanoliposomal cineole at $1-10 \mu \mathrm{g} / \mathrm{mL}$ or $1-40 \mu \mathrm{g} / \mathrm{mL}$ concentration (in nontoxic ranges). The supernatants of cells were then removed and cells were treated with nanoliposomal preparations containing either AsODN or ScODN at $150 \mathrm{nM}$ ODN in the culture medium for 48 hours. After this period, sequence specific-antisense activity of nanoliposomal ODNs was evaluated by MTT. The same experiments were performed in the absence of cineole as control.

2.2.5. Flow Cytometry. Quantitative cellular uptake of liposomes was evaluated by flow cytometry. 300,000 cells were seeded in each well of 6-well plates (NUNC, Denmark). After 24-hour incubation, the cells were transfected for 4 hours by $300 \mathrm{nM}$ FITC-ODN containing liposomes either at $4^{\circ} \mathrm{C}$ or at $37^{\circ} \mathrm{C}$. Cells were cotreated in some wells with sodium azide $(10 \mathrm{mM})$ as active transport inhibitor and in some wells cells were treated with liposomal cineole (1\%). Cells were detached from the plates, suspended in culture medium, and then centrifuged. Cell pellets were then reconstituted in $500 \mu \mathrm{L}$ of either cold PBS or monensin solution $(20 \mu \mathrm{M})$ in PBS. Cells were kept cold (on ice) until the measurement of the cell-associated fluorescence by FACSCalibur dual laser flow cytometer (Becton Dickinson, USA). Cell Quest software (Becton Dickinson, USA) was used for data acquisition through FL1 $(530 \mathrm{~nm})$ and analysis for 10,000 gated events. Cell-associated fluorescence intensities were expressed as the average of these measurements \pm S.E. $(n=3)$.
2.2.6. Fluorescence Microscopy. Transfected cells were fixed with paraformaldehyde and then were permeabilized with Triton X-100 (0.4\%). Subsequently, the nucleus was stained with DAPI $(50 \mu \mathrm{M})$. Cellular internalization of liposomal FITC-ODN was studied by Axiovert fluorescence microscope (Zeiss, Germany) using AxioVision software (Zeiss, Germany).

2.2.7. RT-PCR Assay of Target Gene Expression. Cells were seeded in 6-well plates one day prior to transfection. Total RNA of 6 hours treated cells (with ODNs alone or ODNs + liposomal cineol) was extracted with TRIzol reagent according to the manufacturer's instructions. RNA concentration and purity were determined by UV-spectrophotometry at $260 \mathrm{~nm}$. RNA quality was studied by $1 \%$ agarose gel electrophoresis followed by ethidium bromide staining. Prior to electrophoresis, RNA was treated with DNase and purified with Qiagen RNAeasy mini column. One microgram of total RNA was first reverse-transcribed with $2 \mu \mathrm{L}$ random hexamer to CDNA and then was applied for PCR amplification [30]. PCR amplification was run for GAPDH (glyceraldehyde-3phosphate dehydrogenase), as house-keeping gene, in PCR thermal cycler (MJ Research, MD) [31]. A volume of $10 \mu \mathrm{L}$ PCR reaction for PKC- $\alpha$ along with its respective human GAPDH was loaded into $1 \%$ agarose gel in Tris-borate EDTA (TBE) buffer $0.5 \mathrm{x}$ and amplified segments were detected by ethidium bromide staining. Data were analyzed using UNSCAN-IT software (Silk Scientific, Inc.). The experiment was performed in triplicate.

\subsection{In Vivo Studies}

2.3.1. Tumor Induction in Nude Mice. Female Balb/c nu/nu mice (6 weeks old, weighing $18 \pm 2 \mathrm{~g}$ ) were purchased from Research Center for Science and Technology in Medicine in Tehran University of Medical Sciences (Tehran, Iran) and maintained at an animal facility in a light- and temperaturecontrolled aseptic environment. The handling of mice and experimental procedures were conducted in accordance with the protocols of Ethics Committee of Shaheed Beheshti University of Medical Sciences.

$8 \times 10^{6}$ A549 cells were harvested and resuspended in $200 \mu \mathrm{L}$ of RPMI 1640 media. The cell suspension was injected into the right flank of the mice subcutaneously to induce the initial tumor [38]. Mice were then monitored and checked for tumoral volume every other day until the tumor volumes reached the suitable size of approximately $500 \mathrm{~mm}^{3}$. Tumor size was measured using a caliper across its two perpendicular diameters [39]. The initial tumors were then removed, cut into pieces, and then passaged into other mice by implanting tumor pieces into the right flank of every animal as secondary tumors (xenograft tumors). The growth curve of the tumors and doubling time of their volume were then evaluated using Skipper model [40]. When the secondary tumor volumes reached a mean value of around $100 \mathrm{~mm}^{3}$, the time was considered as time-zero and the mice were randomly assigned to groups of 3 mice for further studies and treatments as described later. 
TABle 1: Properties of prepared oligodeoxy nucleotide- (ODN-) and cineole-containing nanoliposomes.

\begin{tabular}{lccr}
\hline Formulation & Size $(\mathrm{nm})$ & Zeta potential $(\mathrm{mV})$ & Encapsulation efficiency $(\%)$ \\
\hline ODN-containing liposomes & $115 \pm 5.0$ & $0.8 \pm 0.7$ & $87.5 \pm 3.8$ \\
Cineole-containing liposomes & $128 \pm 2.7$ & $6.8 \pm 0.6$ & $67.7 \pm 0.3$ \\
\hline
\end{tabular}

2.3.2. Animal Studies of Antisenses ODNs and Enhancement Effect of Liposomal Cineole. The antitumoral effects of liposomal antisenses and the enhancement effects of cineole on this process were investigated in tumor-bearing mice. Nanoliposomal formulation was applied intratumorally at $0.5 \mathrm{mg}$ ODN $/ \mathrm{kg}$ using approximately $100 \mu \mathrm{L}$ of nanoliposomal suspension containing $100 \mu \mathrm{g} / \mathrm{mL}$ antisense for each mouse. Cineole-containing nanoliposome was used as pretreatment (pretreatment injection was selected based on our finding in lab and comparison with cotreatment) by applying $100 \mu \mathrm{L}$ of nanoliposomal cineole $(80 \mu \mathrm{g} / \mathrm{mL})$ as intratumoral injection 2 hours prior to nanoliposomal ODN application. This process (application of cineole and ODN nanoliposomes) was performed every 4 days for 12 days. Volumes of tumors were measured 2-3 times a week from time zero to 1 month after the first injection.

2.3.3. Hematoxylin and Eosin Staining. At the end of the observation period, mice were sacrificed and tumors were removed, cut, and fixed in $10 \%$ formalin prior to paraffin processing and embedding for further pathological studies. Routine hematoxylin and eosin (HE) staining [41] was carried out on harvested tumor samples. Slides were then observed under light microscope and the images were captured and analyzed for cell death pattern. Based on morphology of cell and nucleus membranes and their content appearance, a portion of dead cells (necrotic or apoptotic cells) in each slide were measured.

2.4. Statistics. Data are presented as mean \pm standard error for 3 replicates unless indicated differently. Linear regression and curve fitting were performed by Graphpad Prism software (San Diego, CA). Statistical comparisons were made using SPSS statistical software (version 18.0) through twosided ANOVA and Tukey's post hoc test. The differences were considered significant when $P<0.05$.

\section{Results}

3.1. Characterization of Liposomes. Prepared ODN-containing liposomes showed a nearly neutral zeta potential $(0.8 \mathrm{mV})$ and size of about $115 \mathrm{~nm}$ (Table 1). These values did not change significantly during 6 months of storage at $4^{\circ} \mathrm{C}(P>0.05)$. The encapsulation efficiency of ODN-containing liposomes was calculated to be more than $87 \%$.

Cineole-containing liposomes showed size and encapsulation efficiency of about $130 \mathrm{~nm}$ and $68 \%$, respectively (Table 1). These particles showed a mild positive charge (about $7 \mathrm{mV}$, Table 1) that is expected to reduce their aggregation and might improve their efficacy [41].

The size of both liposomes used in the present study (115 and $130 \mathrm{~nm}$ ) is close to the optimum size for liposomal drug delivery (around $100 \mathrm{~nm}$ ) [42].

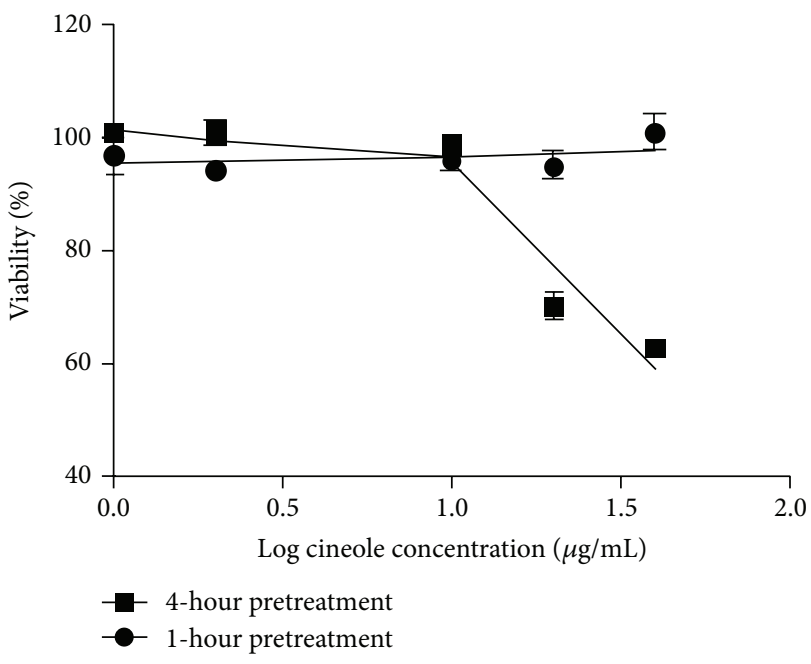

FIGURE 1: Effect of concentration and exposure time on cytotoxicity of nanoliposomal cineole against A549 cells in vitro. Data are presented as mean \pm standard.

3.2. Determination of Cineole Cytotoxicity and Enhancer Concentration. Figure 1 shows the effect of liposomal cineole on the viability of A549 cells in vitro. As is shown, cineole did not affect cell viability for 1-hour pretreatment over the whole concentration range used here ( 1 to $40 \mu \mathrm{g} / \mathrm{mL})$. However, after 4-hour pretreatment, although there is no significant cytotoxicity below $10 \mu \mathrm{g} / \mathrm{mL}$, the viability of cells decreased significantly at higher concentrations of 20 and $40 \mu \mathrm{g} / \mathrm{mL}$. Segmental analysis showed that a linear relationship exists between cell viability and log concentration (Figure 1). The slope of the corresponding line was defined here as antiproliferative-concentration sensitivity (ACS) that was $-60 \pm 5.5$ in the range of $10-40 \mu \mathrm{g} / \mathrm{mL}$ for 4 -hour pretreatment. The slope (ACS) for 1-hour pretreatment $(1.9 \pm$ $0.5)$ and 4-hour pretreatment below $10 \mu \mathrm{g} / \mathrm{mL}(-5.6 \pm 3.1)$ was not significantly different from zero $(P>0.05)$. Therefore, all subsequent cineole-treatment protocols employed nontoxic condition based on the above results.

\subsection{Effect of Cineole on Liposomal ODN Cytotoxicity In Vitro.} Figure 2 provides the effects of different cineole concentrations and treatment protocols on antiproliferative effects of liposomal ODNs. Results showed that control nanoliposomal ODN (ScODN) does not show a significant cytotoxicity in the absence or presence of 1-hour cineole pretreatment over $1-40 \mu \mathrm{g} / \mathrm{mL}$. The relationship between cell viability and log concentration was found to be linear with a slope (ACS) of $(-0.8 \pm 0.7)$ that is not different from zero $(P>0.05)$. When the pretreatment time was increased to 4 hours, the nanoliposomal ScODN showed a slight decrease in the cell viability 


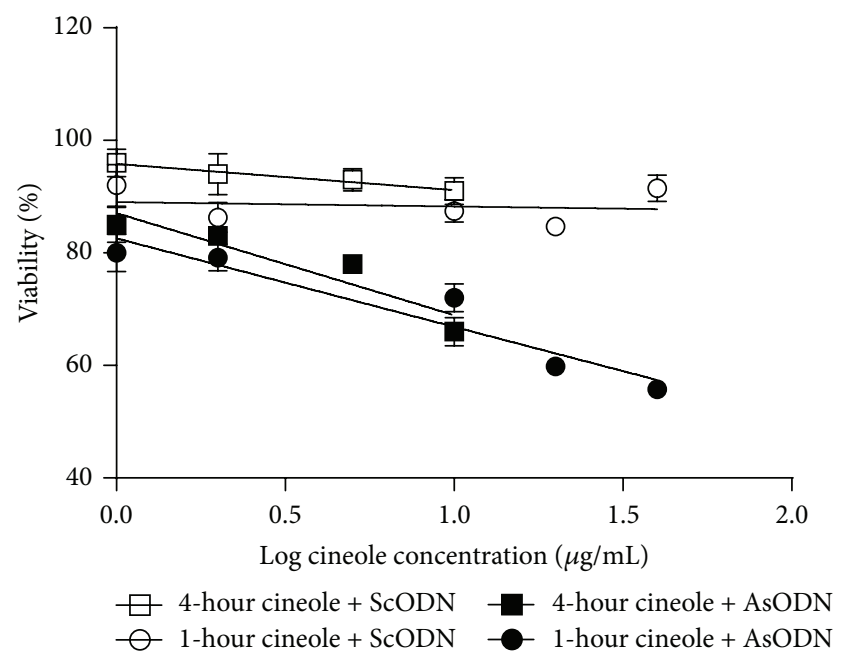

FIGURE 2: Effect of cineole pretreatment on antiproliferative action of nanoliposomal antisense oligonucleotide (AsODN) in comparison to its control $(\mathrm{ScODN})$ at different treatment times. Data are presented as mean \pm standard error $(n=6)$.

upon cineole concentration increase (slope $=-4.7 \pm 0.2)$ and the viability reached to a value of about $91 \%$ at $10 \mu \mathrm{g} / \mathrm{mL}$. These data were used to select nontoxic concentration of cineole to apply in gene delivery experiments.

Nanoliposomal antisense (AsODN) showed a $20 \%$ reduction in cell viability in the absence of cineole that was significant in comparison to ScODN. Cineole treatment caused a concentration-dependent increase in antisense effect for both 1- and 4-hour pretreatment times (Figure 2). The cell viability reached a value of about $70 \%$ at $10 \mathrm{~g} / \mathrm{mL}$ and $55 \%$ at $40 \mu \mathrm{g} / \mathrm{mL}$. The slopes of viability versus concentration line were calculated to be $-15.7 \pm 0.7$ and $-18.1 \pm 1.2$ for $1-$ and 4-hour cineole pretreatments, respectively, indicating that increased pretreatment time causes a slight increase in the sensitivity.

3.4. Fluorescence Microscopy and Flow Cytometry. Figure 3 shows that cationic liposomes can increase the cellular uptake of AsODN very significantly from $9.0 \pm 0.5$ in free ODN to a value of $84.2 \pm 3.9$. Cineole was able to further increase the cell-associated fluorescence of liposomal ODN to a value of $134.3 \pm 6.5(P<0.05)$. In contrast, the effect of cineole on the cellular uptake of free ODN was negligible $(P>0.05)$.

After decreasing the incubation temperature from $37^{\circ} \mathrm{C}$ to $4^{\circ} \mathrm{C}$, cell-associated fluorescence intensity of the SALPs diminished to $22.8 \pm 5.2$. Almost the same effect was observed when the cells were coincubated with sodium azide at $37^{\circ} \mathrm{C}$ (intensity of $39.0 \pm 4.3$ ). Cineole treatment did not change the cellular fluorescence intensity with these inhibitory conditions $\left(4^{\circ} \mathrm{C}\right.$ or in presence of sodium azide). The fluorescent intensities in the presence of cineol at $4^{\circ} \mathrm{C}$ or in the presence of cineole and sodium azide were calculated to be $22.3 \pm 1.7$ and $46.9 \pm 4.7$, respectively that are comparable to the abovementioned values. Incubation at $4^{\circ} \mathrm{C}$ or with sodium azide is expected to block the cellular uptake of the liposomes.

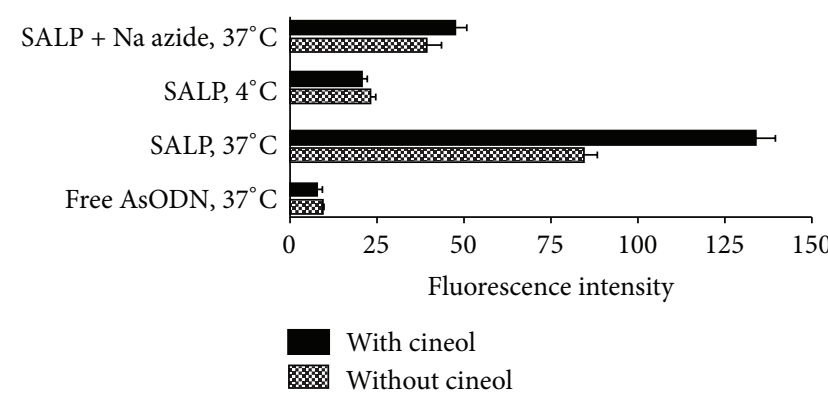

FIGURE 3: Flow cytometric analysis of the cellular uptake of prepared cationic liposomes (SALPs) containing FITC-labelled antisense oligodeoxynucleotides (AsODN) in cineole-treated (dark bars) or untreated (light bars) samples at $37^{\circ} \mathrm{C}$ or $4^{\circ} \mathrm{C}$ in the presence or absence of sodium azide treatments. Data are presented as mean \pm standard error $(n=2)$. Data of untreated samples are from Tamaddon. (From [43] with permission from the Corresponding Author and based on the related Copyright Agreements with the publisher).

These data confirms endocytosis as the main entrance route of liposomal ODNs.

Figure 4 shows that the green fluorescence of FITC-ODN probe is diffusively scattered in the cells transfected with the ODN-containing liposomes (SALPs) (c), while no distinct intracellular fluorescence was observed if the cells incubated with free FITC-ODN at the same concentration (a). Cineole treatment increased the intracellular trafficking of the SALP (d) and the cell-associated fluorescence of FITC-ODN (b).

3.5. RT-PCR Determination of Antisense Activity. Specific PCR products of 172 and 531 base pairs were obtained for PKC- $\alpha$ and GAPDH (internal control), respectively. Treatment with $300 \mathrm{nM}$ AsODN inhibited the gene expression $(39.0 \% \pm 7.1 \%)$ while no obvious inhibition of PKC$\alpha$ was observed with ScODN. Coincubation of the cells with $1 \%$ liposomal cineole at the similar SALP concentration increased the sequence-specific inhibition of the target gene by about 2 times to $82.5 \%$ (Figure $5, P<0.01$ ).

3.6. Effect of Cineole on In Vivo Tumor Growth in Xenograft Model. Tumor growth profile in nude mice (Figure 6(a)) showed that nanoliposomal antisense decreases the tumor volume from 832 to $440 \mathrm{~mm}^{3}$ after 30 days. Nanoliposomal cineole further increased the antisense activity significantly $(P<0.05)$ by decreasing the tumor volume twice more to a value of $205 \mathrm{~mm}^{3}$. Scrambled ODN-containing formulation and liposomal cineole also decreased the tumor volume, but the effect was not that significant $(P>0.05)$ (Figure 6).

Data were also interpreted according to tumor volume doubling time (Figure 6(b)). Tumor volume doubling time in control (nontreated) animals was calculated to be $9.6 \pm$ 0.6 days. Nanoliposomal AsODN increased this doubling time significantly $(P<0.05)$ to a value of 13.4 days. After cineole treatment, the doubling time was further increased $(P<0.01)$ more than twofold to 31.4 days. Liposomal ScODN (negative control) did not change the doubling 

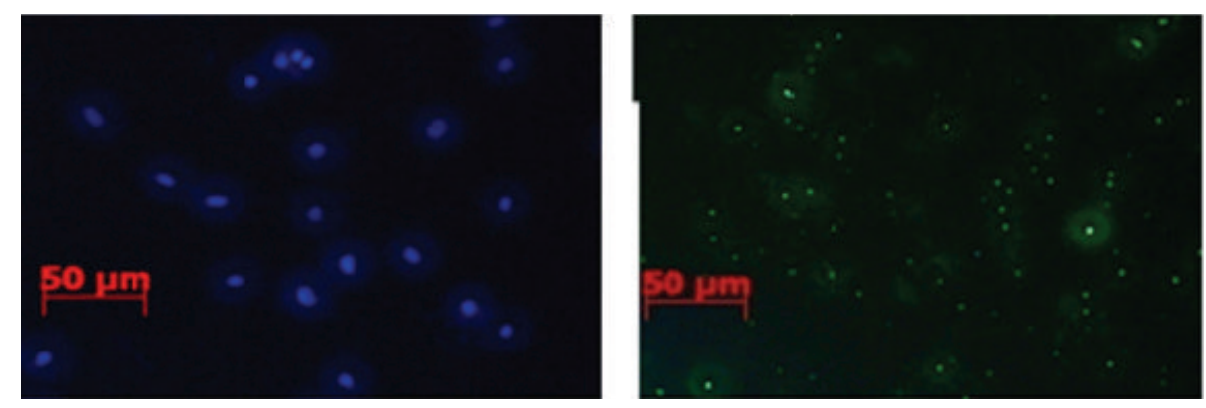

(a)
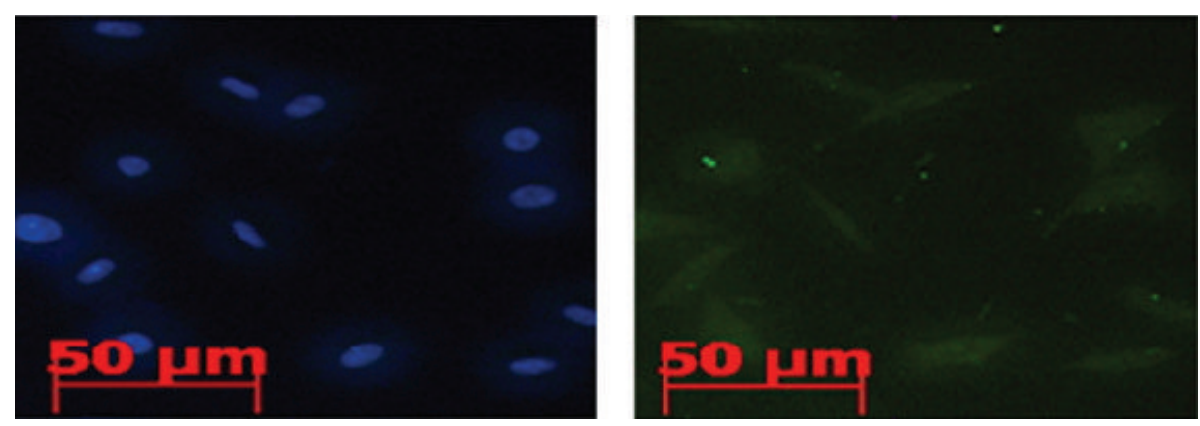

(b)
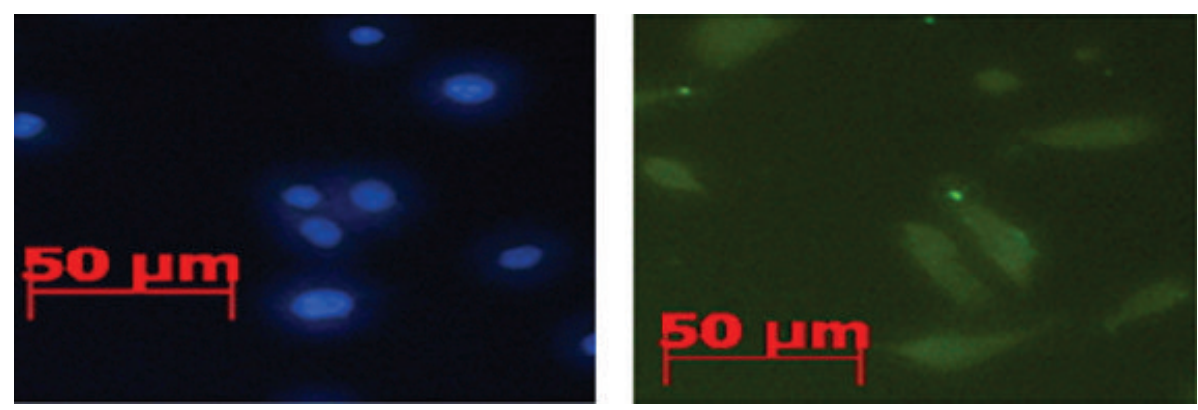

(c)
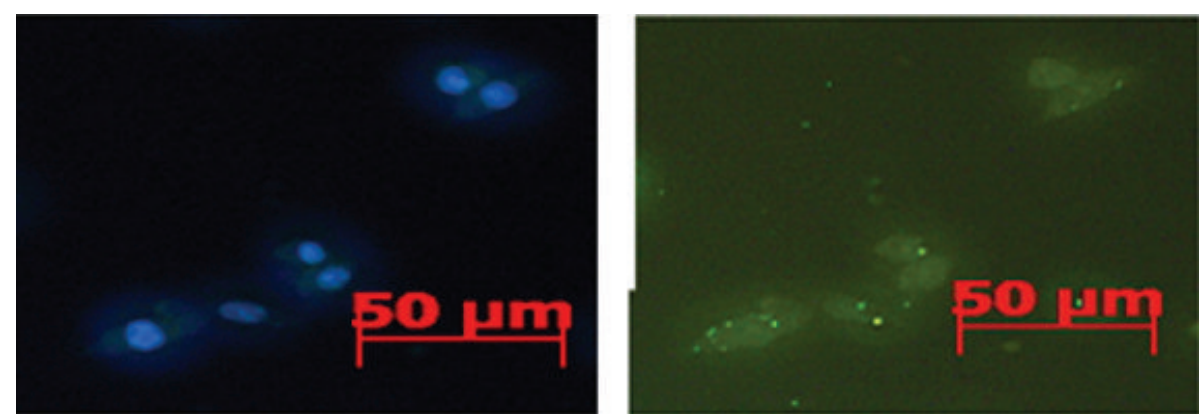

(d)

FIGURE 4: Epifluorescence microscopy study of FITC-antisense oligodeoxynucleotides (AsODN) cellular distribution. Cytoplasmic distribution of fluorescein green fluorescence (right images) was demonstrated after 4-hour incubation with either free AsODN (a, b), liposomal AsODN (c, d), cineole-treated free AsODN (b), and cineole-treated liposomal AsODN (d). Left images illustrate DAPI-stained nucleuses of the same systems.

time indicating that the increased doubling time of AsODN is related to sequence specific inhibitory effect. Liposomal cineole was able to increase the doubling time marginally to 12.3 days.

3.7. Hematoxylin and Eosin Staining. Figure 7 illustrates HEstained images of tumor for different treatments. The severity of cell death was increased upon cineole pretreatment (Figure 7 ) and more than $84 \%$ of cells had died. In the group that was treated with nanoliposomal AsODN alone, visible areas of necrotic and apoptotic cells can be seen in related slide and almost $32.4 \%$ of cells were dead (Figure 7). Only small punctuate necrosis and apoptosis (i.e., less than 3\%) were seen in ScODN-treated group (negative 


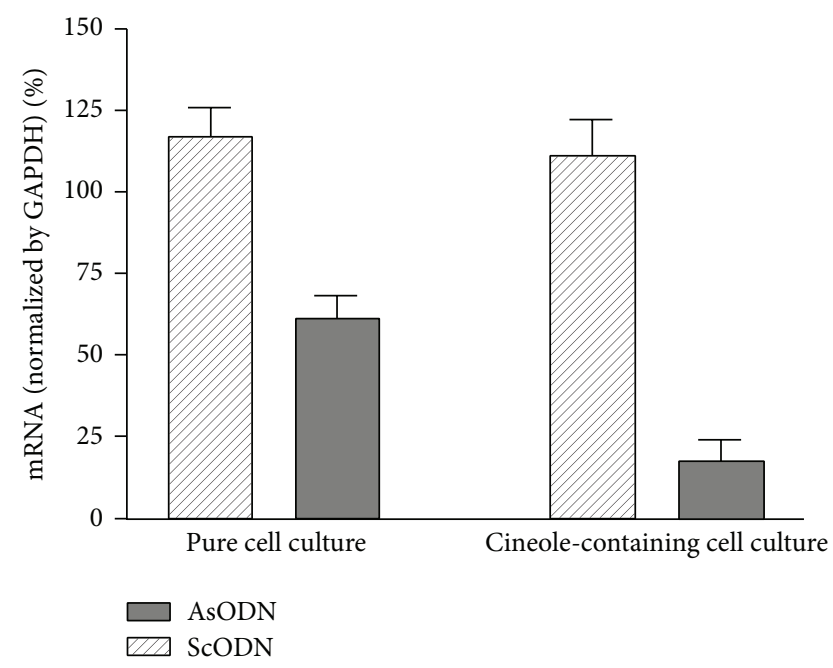

FIGURE 5: RT-PCR determination of protein kinase C- $\alpha$ (PKC- $\alpha$ ) expression. A549 cells were transfected with the liposomal antisense $(\mathrm{As})$ or scrambled $(\mathrm{Sc})$ oligodeoxynucleotides (ODN). Cells were incubated with either pure cell culture medium alone (left bars) or cineole-containing cell culture medium (right bars). Quantitative expressed target mRNA. GAPDH data are provided as control.

control, Figure 7) and no obvious necrosis and apoptosis were observed in A549 tumors of untreated group (Figure 7). Pattern of cell death and debris of dead cell imply that apoptosis is probably responsible for tumor inhibition.

\section{Discussion}

Present cell culture studies showed that applied AsODN can decrease cell viability, with minor effects from scrambled antisense (control), revealing that the effect of applied antisense on cell viability is through specific mRNA inhibition, which is in good correlation with Tamaddon findings [43]. Animal data also revealed that nanoliposomal antisense was able to decrease the tumor volume and increase its doubling time. These findings are in agreement with Yazaki et al. who showed that intraperitoneal administration of phosphorothioate antisense oligonucleotides against PKCalpha mRNA were able to inhibit xenografted glial tumors in nude mice [44] and that of Ying et al. who showed tumor inhibition effect of Elk-1 (an antisense ODN against PKCalpha) in carcinoma, both in vitro and in nude mice [45].

Cineole pretreatment increased the antisense effect significantly by decreasing the tumor volume and increasing its doubling time (more than 200\%) or decreasing cell viability in cell culture. It has been shown that endocytosis and interactions of liposomes with cytoplasmic and endosomal membranes are the main mechanisms for liposomal antisense delivery $[9,43]$. On the other hand, cineole performs its enhancement effect toward small molecules through either complexation with small polar molecules [23] or lipid bilayer fluidization $[22,46]$. Between these two mechanisms, the importance of complexation might be lower due to the mechanism of entrance of these particles into the cell in liposomal delivery. Therefore, it might be concluded here that cineole exerts its action through lipid bilayers fluidization. Such a fluidization can increase membrane fusion or disruption at liposomes, cell membrane, and/or endosome levels and, therefore, improve uptake of liposomes into the cell and/or increase their endosomal escape. This proposed mechanism is in agreement with increased deformability and higher cutaneous uptake of cineole-containing liposomes in comparison to control liposomes [47]. In this direction, it has been shown that presence of $40 \mathrm{~mol} \%$ DOPE (a lipid fluidizer) causes enhanced transfection efficiency of Gal lipoplex in Hela cells [48]. The main action of DOPE is enhancing fusion by producing hexagonal phases; therefore DOPE decreases the stability of liposomes due to its geometry and it has been shown that in vivo application of DOPE is limited because of its interaction with blood cells [49] and instability of the liposomes [18]. It has also been shown that partially substituted polylysine (bilayer destabilizer) causes a dramatic increase by (3-4.5 orders of magnitude) of the transfection efficiency of DNA/polylysine polyplexes [50].

Our results also show that the enhancement effect of cineole is concentration-dependent, which is in good agreement with concentration-dependent enhancement effect of cineole toward permeation of 5-fluorouracil and oestradiol through a lamellar liquid crystalline lipid model structure [26]. In comparison to urea as penetration enhancer for gene delivery cineol could improve transfection efficiency of liposomal ODN by around 2-fold more intensive [51]. Results also showed that treatment time affects enhancement effect of cineole, but its intensity was found to be less than cineole concentration (Figure 2).

The present results also indicate that cell culture data correlate well with animal studies in terms of enhancement effect, indicating that cell culture studies might be used for screening of enhancers against liposomal delivery.

Besides enhancement effects, cineole showed cytotoxic effect against A549 cells at high concentrations and/or long treatment times. This is in agreement with Bowen and Ali who showed that some terpenes may show antiproliferative effect in cancer cells [52].

Although the data obtained clearly indicates that cineole has the ability to enhance the cellular delivery of liposomal AsODNs and also improves their antitumor efficacy, we assumed that at least the in vitro results should be extended to a wider panel of cell lines to substantiate the conclusion that cineole increase liposomal AsODNs delivery and antitumor effects. Therefore, these steps were also implemented for OV2008 (ovarian carcinoma) and A431 (epidermoid carcinoma) as two control cell lines; and results (have not been demonstrated here) also confirmed the above conclusion.

To conclude, the present results clearly show that cineole and possibly other terpenes and chemical penetration enhancers are able to increase nanoliposomal AsODN delivery and its antitumoral effect. In terms of drug delivery, increased permeation means either more pharmacological effect or less drug content for the same effect, both of which will result in more economic systems for expensive treatment methods like liposomal gene delivery. In this direction, Dempsey et al. have shown that sublethal doses of chemotherapeutic agents can be combined with membrane 


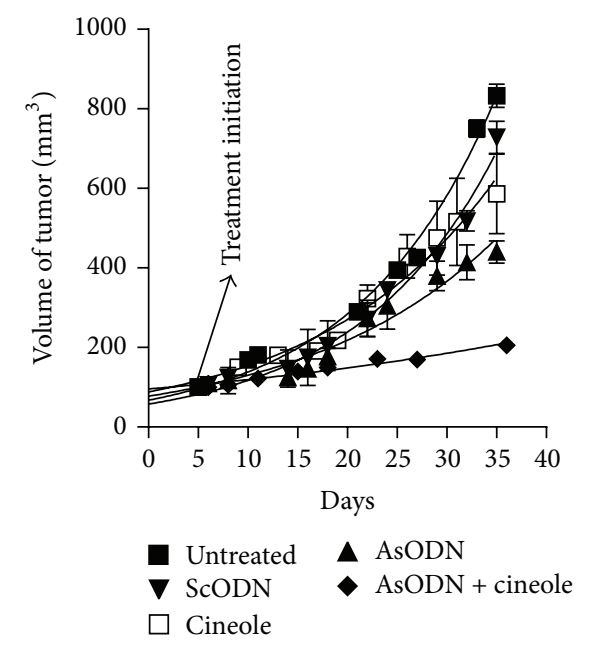

(a)
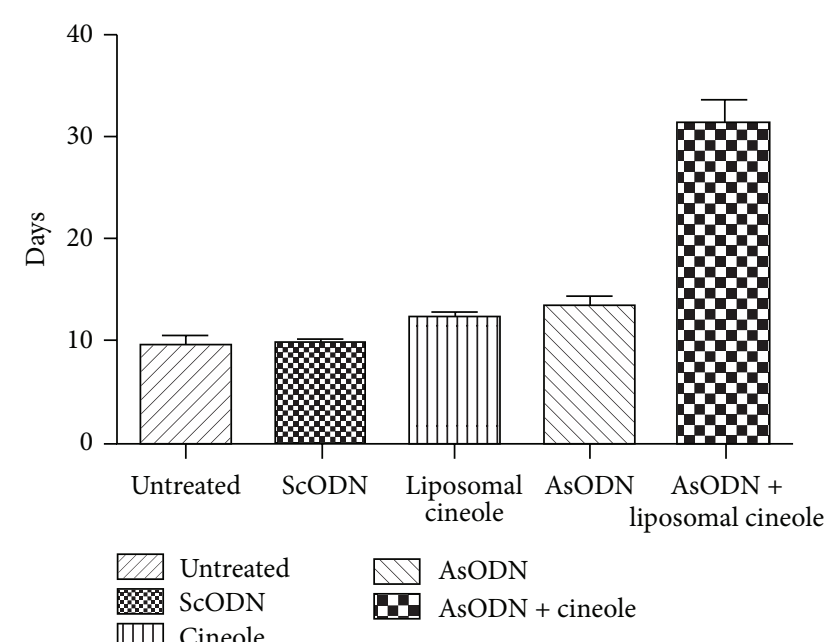

(b)

FIGURE 6: Tumor growth profile (a) and doubling time (b) as indicators of inhibitory effects of different antisense (As) or scrambled (Sc) oligodeoxynucleotides (ODN) nanoliposomal formulations in the presence or absence of cineole in nude mice in comparison to untreated control animal. Data are presented as mean \pm standard error $(n=3)$.
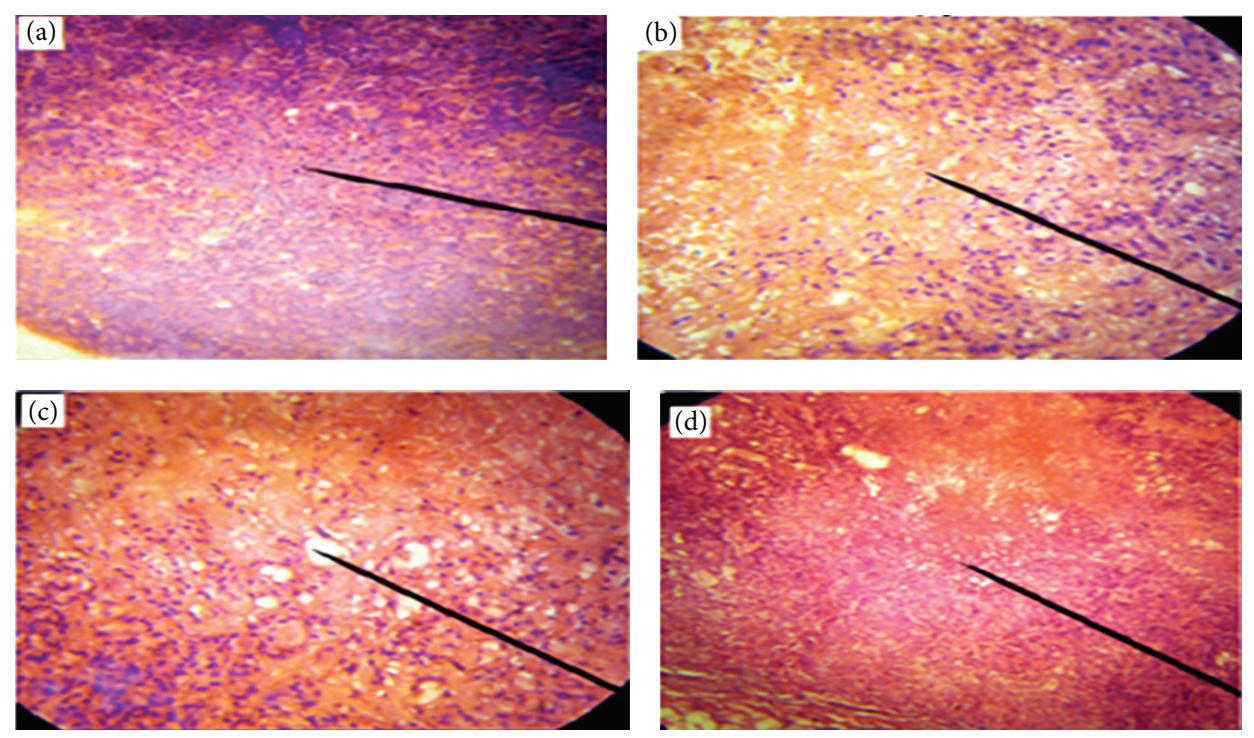

FIGURE 7: Hematoxylin/eosin staining (parallel to Annexin Kit selection) of untreated tumor (a) and tumor treated by nanoliposomal antisense $(b, c)$ or scrambled (d) oligonucleotides in the absence $(b, d)$ or presence $(c)$ of cineole.

fluidizing treatments (hyperthermia, benzyl alcohol, and ethanol) to produce a significant increase in drug efficacy and apoptosis [53]. Other enhancement methods are also reported for enhancement of liposomal or nonliposomal ODN delivery like sonophoresis [20], electroporation, complex formation, or radiation $[54,55]$. In comparison to such methods, chemical penetration enhancers are considered less invasive, readily available, easy to apply, and less expensive.

\section{Conclusion}

Present investigation clearly shows that cineole and possibly other membrane fluidizing agents or penetration enhancers can improve antitumor activity of nanoliposomal antisense oligonucleotides, possibly through improved cellular delivery and cytoplasmic release. Although several other enhancement methods are available, such chemical enhancement might provide more versatile, less expensive, and noninvasive approaches for gene delivery. This strategy can open new era in the field of liposomal gene therapy. Further investigations including intravenous applicability of cineolefluidized liposome in animal models and application of other biocompatible membrane fluidizing agents are suggested and are in progress in our laboratories.

\section{Conflict of Interests}

The authors declare that there is no conflict of interests regarding the publication of this paper. 


\section{Acknowledgments}

This work was supported by Pharmaceutical Sciences Research Centre at Shahid Beheshti University of Medical Sciences (SBMU), Tehran, Iran (Grant no. 400.8332) to Hamid Reza Moghimi. This work is from Ph.D. theses of Mostafa Saffari at School of Pharmacy, SBMU.

\section{References}

[1] N. Dias and C. A. Stein, "Antisense oligonucleotides: basic concepts and mechanisms," Molecular Cancer Therapeutics, vol. 1, no. 5, pp. 347-355, 2002.

[2] B. Yang, J. Gao, Z. Rao, B. Zhang, W. Ouyang, and C. Yang, "Antisense oligonucleotide targeting matrix metalloproteinase7 (MMP-7) changes the ultrastructure of human A549 lung adenocarcinoma cells," Ultrastructural Pathology, vol. 35, no. 6, pp. 256-259, 2011.

[3] S. Akhtar, M. D. Hughes, A. Khan et al., "The delivery of antisense therapeutics," Advanced Drug Delivery Reviews, vol. 44, no. 1, pp. 3-21, 2000.

[4] N. S. Templeton, D. D. Lasic, P. M. Frederik, H. H. Strey, D. D. Roberts, and G. N. Pavlakis, "Improved DNA: liposome complexes for increased systemic delivery and gene expression," Nature Biotechnology, vol. 15, no. 7, pp. 647-652, 1997.

[5] F. Pastorino, D. Stuart, M. Ponzoni, and T. M. Allen, "Targeted delivery of antisense oligonucleotides in cancer," Journal of Controlled Release, vol. 74, no. 1-3, pp. 69-75, 2001.

[6] S. C. Semple, S. K. Klimuk, T. O. Harasym et al., "Efficient encapsulation of antisense oligonucleotides in lipid vesicles using ionizable aminolipids: formation of novel small multilamellar vesicle structures," Biochimica et Biophysica Acta, vol. 1510, no. 1-2, pp. 152-166, 2001.

[7] D. S. Friend, D. Papahadjopoulos, and R. J. Debs, "Endocytosis and intracellular processing accompanying transfection mediated by cationic liposomes," Biochimica et Biophysica Acta, vol. 1278, no. 1, pp. 41-50, 1996.

[8] Y. Xu and F. C. Szoka Jr., "Mechanism of DNA release from cationic liposome/DNA complexes used in cell transfection," Biochemistry, vol. 35, no. 18, pp. 5616-5623, 1996.

[9] G. Sahay, D. Y. Alakhova, and A. V. Kabanov, "Endocytosis of nanomedicines," Journal of Controlled Release, vol. 145, no. 3, pp. 182-195, 2010.

[10] H. Harashima, Y. Shinohara, and H. Kiwada, "Intracellular control of gene trafficking using liposomes as drug carriers," European Journal of Pharmaceutical Sciences, vol. 13, no. 1, pp. 85-89, 2001.

[11] J. Mönkkönen and A. Urtti, "Lipid fusion in oligonucleotide and gene delivery with cationic lipids," Advanced Drug Delivery Reviews, vol. 34, no. 1, pp. 37-49, 1998.

[12] G. L. Lukacs, P. Haggie, O. Seksek, D. Lechardeur, N. Freedman, and A. S. Verkman, "Size-dependent DNA mobility in cytoplasm and nucleus," The Journal of Biological Chemistry, vol. 275, no. 3, pp. 1625-1629, 2000.

[13] M. B. Bally, P. Harvie, F. M. P. Wong, S. Kong, E. K. Wasan, and D. L. Reimer, "Biological barriers to cellular delivery of lipidbased DNA carriers," Advanced Drug Delivery Reviews, vol. 38, no. 3, pp. 291-315, 1999.

[14] F. Shi and D. Hoekstra, "Effective intracellular delivery of oligonucleotides in order to make sense of antisense," Journal of Controlled Release, vol. 97, no. 2, pp. 189-209, 2004.
[15] M. C. De Oliveira, E. Fattal, C. Ropert, C. Malvy, and P. Couvreur, "Delivery of antisense oligonucleotides by means of pH-sensitive liposomes," Journal of Controlled Release, vol. 48, no. 2-3, pp. 179-184, 1997.

[16] R. Rennert, C. Wespe, A. G. Beck-Sickinger, and I. Neundorf, "Developing novel hCT derived cell-penetrating peptides with improved metabolic stability," Biochimica et Biophysica Acta, vol. 1758, no. 3, pp. 347-354, 2006.

[17] I. M. Hafez, S. Ansell, and P. R. Cullis, "Tunable pH-sensitive liposomes composed of mixtures of cationic and anionic lipids," Biophysical Journal, vol. 79, no. 3, pp. 1438-1446, 2000.

[18] S. W. Hui, M. Langner, Y.-L. Zhao, P. Ross, E. Hurley, and K. Chan, "The role of helper lipids in cationic liposome-mediated gene transfer," Biophysical Journal, vol. 71, no. 2, pp. 590-599, 1996.

[19] L. Y. Song, Q. F. Ahkong, Q. Rong et al., "Characterization of the inhibitory effect of PEG-lipid conjugates on the intracellular delivery of plasmid and antisense DNA mediated by cationic lipid liposomes," Biochimica et Biophysica ActaBiomembranes, vol. 1558, no. 1, pp. 1-13, 2002.

[20] Y.-K. Luo, Y.-Z. Zhao, C.-T. Lu, J. Tang, and X.-K. Li, “Application of ultrasonic gas-filled liposomes in enhancing transfer for breast cancer-related antisense oligonucleotides: an experimental study," Journal of Liposome Research, vol. 18, no. 4, pp. 341-351, 2008.

[21] A. C. Williams, H. G. M. Edwards, E. E. Lawson, and B. W. Barry, "Molecular interactions between the penetration enhancer 1,8-cineole and human skin," Journal of Raman Spectroscopy, vol. 37, no. 1-3, pp. 361-366, 2006.

[22] H. R. Moghimi, A. C. Williams, and B. W. Barry, "A lamellar matrix model for stratum corneum intercellular lipids. V. Effects of terpene penetration enhancers on the structure and thermal behaviour of the matrix," International Journal of Pharmaceutics, vol. 146, no. 1, pp. 41-54, 1997.

[23] H. R. Moghimi, A. C. Williams, and B. W. Barry, "Enhancement by terpenes of 5 -fluorouracil permeation through the stratum corneum: model solvent approach," Journal of Pharmacy and Pharmacology, vol. 50, no. 9, pp. 955-964, 1998.

[24] H. R. Moghimi, A. C. Williams, and B. W. Barry, "A lamellar matrix model for stratum corneum intercellular lipids. IV. Effects of terpene penetration enhancers on the permeation of 5-fluorouracil and oestradiol through the matrix," International Journal of Pharmaceutics, vol. 145, no. 1-2, pp. 49-59, 1996.

[25] A. C. Williams and B. W. Barry, "Penetration enhancers," Advanced Drug Delivery Reviews, vol. 56, no. 5, pp. 603-618, 2004.

[26] H. R. Moghimi, A. C. Williams, and B. W. Barry, "A lamellar matrix model for stratum corneum intercellular lipids IV. Effects of terpene penetration enhancers on the permeation of 5-fluorouracil and oestradiol through the matrix," International Journal of Pharmaceutics, vol. 145, no. 1-2, pp. 49-59, 1996.

[27] Z. S. Saify, O. Ahsan, and A. Dayo, "Cineole as skin penetration enhancer," Pakistan Journal of Pharmaceutical Sciences, vol. 13, no. 1, pp. 29-32, 2000.

[28] A. Ahad, M. Aqil, K. Kohli, Y. Sultana, M. Mujeeb, and A. Ali, "Interactions between novel terpenes and main components of rat and human skin: mechanistic view for transdermal delivery of propranolol hydrochloride," Current Drug Delivery, vol. 8, no. 2, pp. 213-224, 2011.

[29] J. L. Anjos, D. de Sousa Neto, and A. Alonso, "Effects of 1,8cineole on the dynamics of lipids and proteins of stratum 
corneum," International Journal of Pharmaceutics, vol. 345, no. 1-2, pp. 81-87, 2007.

[30] C. M. Heard, D. Kung, and C. P. Thomas, "Skin penetration enhancement of mefenamic acid by ethanol and 1,8-cineole can be explained by the 'pull' effect," International Journal of Pharmaceutics, vol. 321, no. 1-2, pp. 167-170, 2006.

[31] H.-F. Song, Z.-M. Tang, S.-J. Yuan, B.-Z. Zhu, and X.-W. Liu, "Antisense candidates against protein kinase C- $\alpha$ designed based on phylogenesis and simulant structure of mRNA," Acta Pharmacologica Sinica, vol. 24, no. 3, pp. 269-276, 2003.

[32] N. Maurer, K. F. Wong, H. Stark et al., "Spontaneous entrapment of polynucleotides upon electrostatic interaction with ethanoldestabilized cationic liposomes," Biophysical Journal, vol. 80, no. 5, pp. 2310-2326, 2001.

[33] E. G. Bligh and W. J. Dyer, "A rapid method of total lipid extraction and purification," Canadian Journal of Biochemistry and Physiology, vol. 37, no. 8, pp. 911-917, 1959.

[34] J. C. M. Stewart, "Colorimetric determination of phospholipids with ammonium ferrothiocyanate," Analytical Biochemistry, vol. 104, no. 1, pp. 10-14, 1980.

[35] W. Wolfgang and S. S. Ulrike, Eds., Gene Therapy of Cancer: Methods and Protocols, vol. 542 of Methods in Molecular Biology, Humana Press, New York, NY, USA, 2nd edition, 2009.

[36] C. Sinico, A. De Logu, F. Lai et al., "Liposomal incorporation of Artemisia arborescens L. essential oil and in vitro antiviral activity," European Journal of Pharmaceutics and Biopharmaceutics, vol. 59, no. 1, pp. 161-168, 2005.

[37] T. Mosmann, "Rapid colorimetric assay for cellular growth and survival: application to proliferation and cytotoxicity assays," Journal of Immunological Methods, vol. 65, no. 1-2, pp. 55-63, 1983.

[38] S. Hou, Z. Zhao, F. Yan et al., "Genetic transfer of PNAS-4 induces apoptosis and enhances sensitivity to gemcitabine in lung cancer," Cell Biology International, vol. 33, no. 3, pp. 276$282,2009$.

[39] I.-Y. Kim, Y.-S. Kang, D. S. Lee et al., "Antitumor activity of EGFR targeted $\mathrm{pH}$-sensitive immunoliposomes encapsulating gemcitabine in A549 xenograft nude mice," Journal of Controlled Release, vol. 140, no. 1, pp. 55-60, 2009.

[40] E. J. Freireich, E. A. Gehan, D. P. Rall, L. H. Schmidt, and H. E. Skipper, "Quantitative comparison of toxicity of anticancer agents in mouse, rat, hamster, dog, monkey, and man," Cancer Chemotherapy Reports, vol. 50, no. 4, pp. 219-244, 1966.

[41] R. Zhao, W. Yang, Z. Wang, G. Li, W. Qin, and J. Wang, "Treatment of transplanted tumor of lung adenocarcinoma A549 transfected by human somatostatin receptor subtype 2 (hsstr2) gene with ${ }^{188}$ Re-RC-160," Nuclear Medicine and Biology, vol. 37, no. 8, pp. 977-987, 2010.

[42] D. C. Drummond, O. Meyer, K. Hong, D. B. Kirpotin, and D. Papahadjopoulos, "Optimizing liposomes for delivery of chemotherapeutic agents to solid tumors," Pharmacological Reviews, vol. 51, no. 4, pp. 691-743, 1999.

[43] A. M. Tamaddon, Effect of some lipid bilayer-destabilizers on release of antisense oligodeoxynucleotides from cationic liposomes and their cytotoxicity [Ph.D. thesis], Shahid Beheshti University of Medical Sciences, Tehran, Iran, 2006.

[44] T. Yazaki, S. Ahmad, A. Chahlavi et al., "Treatment of glioblastoma $\mathrm{U}-87$ by systemic administration of an antisense protein kinase C- $\alpha$ phosphorothioate oligodeoxynucleotide," Molecular Pharmacology, vol. 50, no. 2, pp. 236-242, 1996.
[45] T.-H. Ying, Y.-H. Hsieh, Y.-S. Hsieh, and J.-Y. Liu, "Antisense oligonucleotide Elk-1 suppresses the tumorigenicity of human hepatocellular carcinoma cells," Cell Biology International, vol. 32, no. 2, pp. 210-216, 2008.

[46] P. A. Cornwell, B. W. Barry, J. A. Bouwstra, and G. S. Gooris, "Modes of action of terpene penetration enhancers in human skin; differential scanning calorimetry, small-angle $\mathrm{X}$-ray diffraction and enhancer uptake studies," International Journal of Pharmaceutics, vol. 127, no. 1, pp. 9-26, 1996.

[47] S. Mura, M. Manconi, C. Sinico, D. Valenti, and A. M. Fadda, "Penetration enhancer-containing vesicles (PEVs) as carriers for cutaneous delivery of minoxidil," International Journal of Pharmaceutics, vol. 380, no. 1-2, pp. 72-79, 2009.

[48] M. D. Kearns, Y. N. Patel, and M. Savva, "Physicochemical characteristics associated with transfection of cationic cholesterolbased gene delivery vectors in the presence of DOPE," Chemistry and Physics of Lipids, vol. 163, no. 8, pp. 755-764, 2010.

[49] F. Sakurai, T. Nishioka, H. Saito et al., "Interaction between DNA-cationic liposome complexes and erythrocytes is an important factor in systemic gene transfer via the intravenous route in mice: the role of the neutral helper lipid," Gene Therapy, vol. 8, no. 9, pp. 677-686, 2001.

[50] P. Midoux, C. Pichon, J.-J. Yaouanc, and P.-A. Jaffrès, "Chemical vectors for gene delivery: a current review on polymers, peptides and lipids containing histidine or imidazole as nucleic acids carriers," British Journal of Pharmacology, vol. 157, no. 2, pp. 166-178, 2009.

[51] M. Saffari, A. M. Tamaddon, F. H. Shirazi, M. A. Oghabian, and H. R. Moghimi, "Improving cellular uptake and in vivo tumor suppression efficacy of liposomal oligonucleotides by urea as a chemical penetration enhancer," Journal of Gene Medicine, vol. 15, no. 1, pp. 12-19, 2013.

[52] I. D. Bowen and Y. A. Ali, "Anti-Tumour Terpene Compounds," United States Patents, 20070259056, 2007.

[53] N. C. Dempsey, H. E. Ireland, C. M. Smith, C. F. Hoyle, and J. H. H. Williams, "Heat Shock Protein translocation induced by membrane fluidization increases tumor-cell sensitivity to chemotherapeutic drugs," Cancer Letters, vol. 296, no. 2, pp. 257-267, 2010.

[54] D. G. Spiller, R. V. Giles, J. Grzybowski, D. M. Tidd, and R. E. Clark, "Improving the intracellular delivery and molecular efficacy of antisense oligonucleotides in chronic myeloid leukemia cells: a comparison of streptolysin-O permeabilization, electroporation, and lipophilic conjugation," Blood, vol. 91, no. 12, pp. 4738-4746, 1998.

[55] R. A. Abela, J. Qian, L. Xu, T. S. Lawrence, and M. Zhang, "Radiation improves gene delivery by a novel transferrinlipoplex nanoparticle selectively in cancer cells," Cancer Gene Therapy, vol. 15, no. 8, pp. 496-507, 2008. 

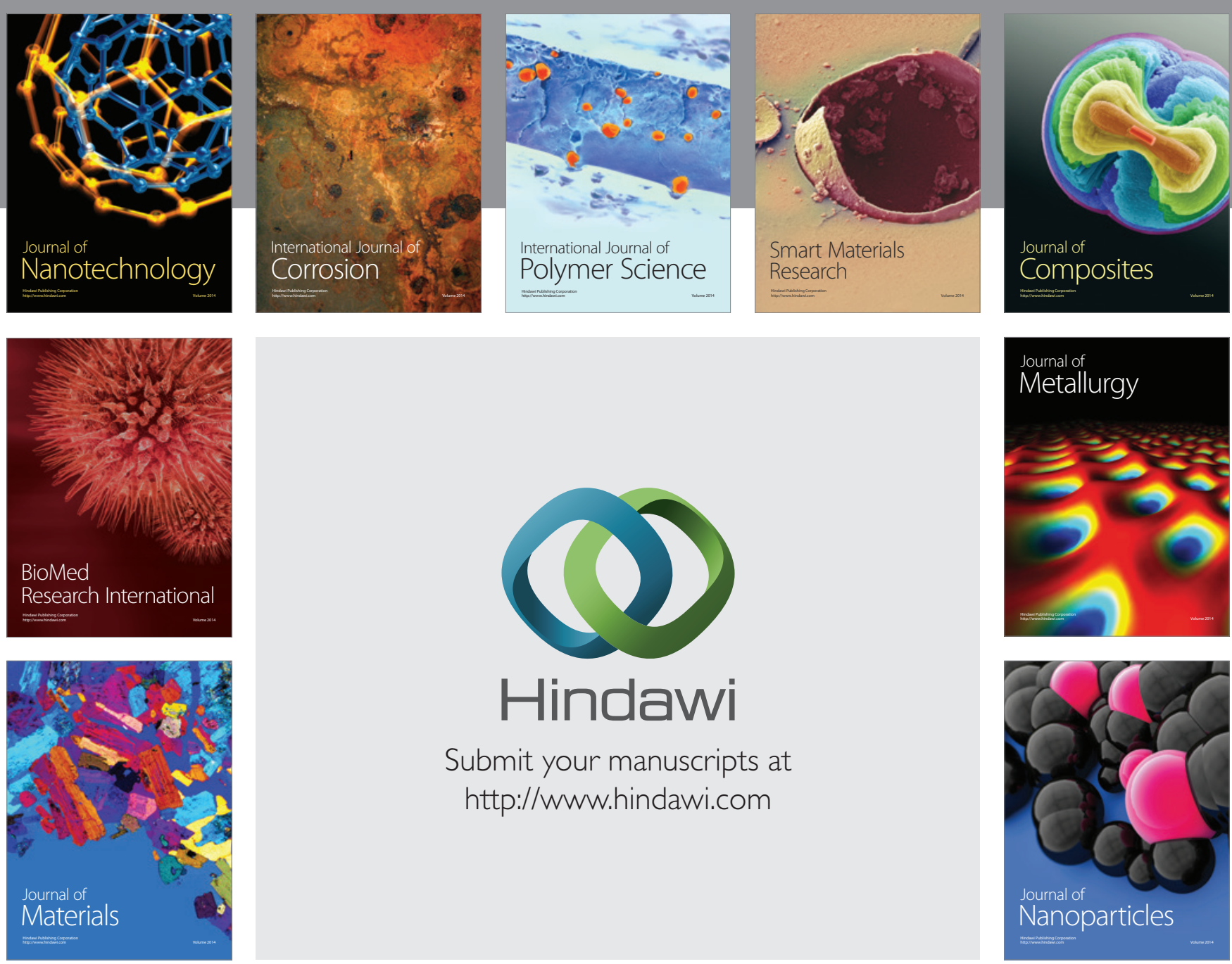

Submit your manuscripts at http://www.hindawi.com
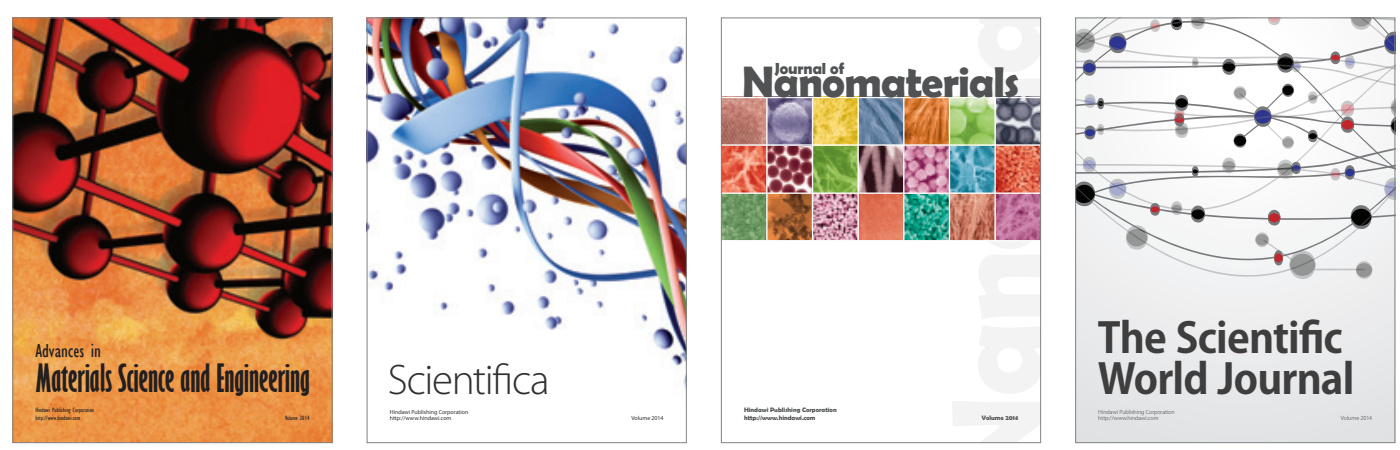

\section{The Scientific World Journal}
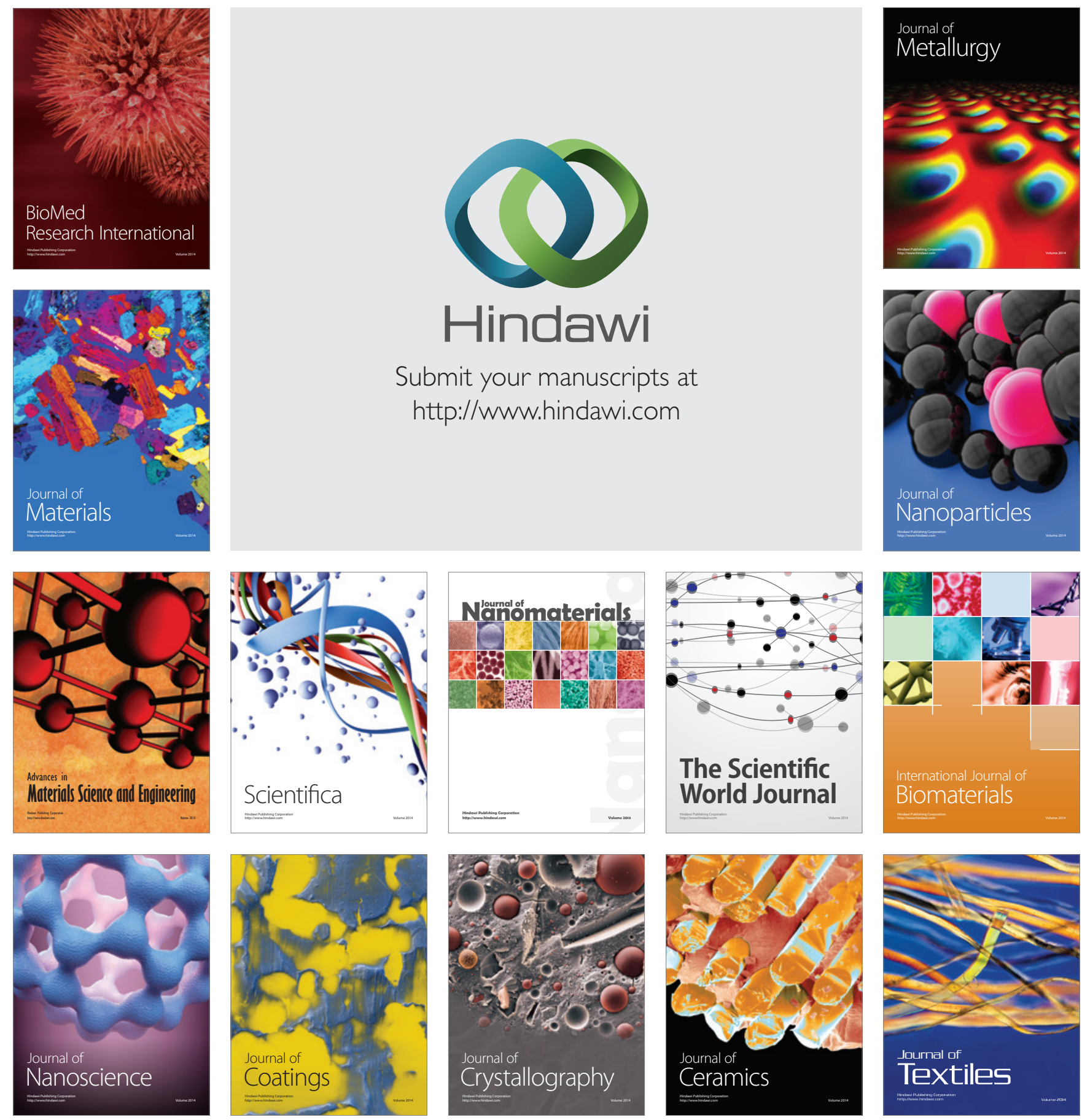